\title{
MEA-DSR: A Multipath Energy-aware Routing Protocol for Wireless Ad Hoc Networks
}

\author{
Floriano De Rango, Paola Lonetti, Salvatore Marano \\ D.E.I.S. Department University of Calabria \\ \{derango, marano\}@deis.unical.it
}

\begin{abstract}
This paper presents a novel multipath energy aware routing for wireless ad hoc network. A deep analysis of different routing metrics such as MBCR, MMBCR and MDR have been led out and the Minimum Drain Rate metric has been selected as energy metric to integrate in the Multipath DSR protocol. Performance comparison with an energy efficient DSR (DSR-MDR) has been presented showing the benefits of the multiple route selection. An update mechanism and a simple data packet scheduling among the energy efficient paths have also been implemented to update the source route cache and for improving the traffic and energy load balancing. Comparison of Multipath DSR with MDR, cache update and round robin scheduling (MEA-DSR) has been also compared with Multipath DSR with MDR metric without cache update mechanism (MDSR-MDR). Simulation results confirm the improvements associated to multipath extension with energy aware metric with respect to the MDR-DSR (unipath routing).
\end{abstract}

"Keywords" Multi-path routing, Ad Hoc Networks, Energy, MDR.

\section{INTRODUCTION}

Numerous studies in literature have focused on multi-path routing, proposing different solutions and ways to see and solve the problem [2-18]. The multi-routes between a source and a destination can be used to transmit the information over all the paths at the same time in order to maximize the flow of data info as well as to split the bandwidth request of a flow over multi-paths in order to increase the success rate of the bandwidth request [2]. Another approach with a different purpose is to use the multi-routes not at the same time, but where one route is used as the primary route and the others as back-up routes in order to reduce the number of route recoveries [5]. Both of these approaches are improvements over uni-path routing protocols, because they have greater resilience to the host mobility in comparison with the relative uni-path version, reducing the delay and increasing the throughput. There are also some multi-path routing protocols that reduce the routing overhead through a single route discovery process able to build more links or node-disjoint routes towards the destination, such as Ad Hoc On Demand Multi-path Distance Vector Routing [10].

In literature there are a lot of works about multipath and power-aware routing protocol. The basic idea of this work is to join these two concepts in a unique protocol: MEA-DSR (Multipath Energy Aware DSR). The MEA-DSR is an extension

Please use the following format when citing this chapter:

De Rango, F., I conetti, P., Marano, S., 2008, in IFIP International Federation for Information Processing, Volume 265, Advances in Ad I loc Networking, eds. Cuenca, P., Gucrero C., Puigjaner, R., Scrra, B., (Boston: Springer), pp. 215225 . 
to the DSR protocol for computing multiple node-disjoint paths [8], where the "best" path is the most energy-efficient. We chose DSR as unipath routing protocol to extend, because it can be suitable from an energetic point of view for its reactive nature [6]. In multipath extension of DSR (Multipath DSR) Route Discovery mechanism of DSR was modified to implement a multipath and energyaware routing. Moreover, a Caching Update Mechanism through probe packets was included to have always updated information in routing cache and a simple round robin data scheduling among multiple selected routes is also implemented in order to balance the traffic load and the energy consumption.

The paper is organized as follows: section II gives a brief overview of multipath routing in MANET; the adopted energy model and metric is introduced in section III; the energy extension to Multipath DSR is explained in section IV; at the end performance evaluation and conclusions are presented in section V and VI.

\section{RELATED WORK}

In the literature, there is much research on multi-path routing for ad hoc networks [2-5, 7-18]; in [2] there can be found a useful overview of this research activity. Multi-path routing offers the advantages of reducing the number of route discovery processes and the end-to-end data packet delay, while increasing the data packet throughput. There are several philosophies that approach the problem of multi-path in a different way. One of them is to use the multi-path routing to make load balancing between the paths [3]; another approach, in the case of QoS support, is to split the bandwidth request among more paths towards the destination in order to increase the success rate of finding routes in the route discovery phase, thus offering QoS guarantees. Multipath routing using a cross-layering approach have also been proposed such as in [10].

In [4] Lee and Gerla propose a multi-path routing algorithm called Split Multipath Routing (SMR) that represents an extension of the Dynamic Source Routing protocol. SMR uses two link-disjoint paths where the traffic is split. The traffic is distributed between the two paths through a per-packet allocation technique. The proposed scheme outperforms DSR because the multi-path routes provide robustness to mobility. The benefits of multi-path are more evident for high mobility speed. Also in [4] an extension of DSR to the multipath case has been proposed. However, in this paper, among many proposed routing schemes, we selected the work proposed by authors in [14] which has been shown to be very efficient and it was extended to the multipath case, investigating the performance of the protocol and energy load balancing under different network scenarios.

\section{Energy-AWARE MOdel AND MDR Metric}

In this section some details about the energy model adopted in accordance with [12], and the energy aware metric will be given. 


\section{Energy model}

A generic expression to calculate the energy required to transmit a packet $p$ is: $E(p)=i \cdot v \cdot t_{p}$ Joules, where: $i$ is the current consumption, $v$ is the voltage used, and $t_{p}$ the time required to transmit the packet. It is supposed that all mobile devices are equipped with IEEE $802.1 \mathrm{lb}$ network interface cards (NICs). The energy consumption values were obtained by comparing commercial products with the experimental data reported in [12].

The values used for the voltage and the packet transmission time were: $v=5 \mathrm{~V}$ and $t_{p}=\left(\frac{p_{h}}{6 \cdot 10^{6}}+\frac{p_{d}}{54 \cdot 10^{6}}\right) \mathrm{s}$, where $p_{h}$ and $p_{d}$ are the packet header and payload size in bits, respectively.

We calculated the energy required to transmit and receive a packet $p$ by using: $E_{t x}(p)=\left(280 \mathrm{~mA} \cdot v \cdot t_{p}\right)$ and $E_{r x}(p)=\left(240 \mathrm{~mA} \cdot v \cdot t_{p}\right)$ respectively. Since receiving a packet and just being idle, i.e., when simply powered on, are energetically similar [15], we assumed $E_{\text {idle }}(p)=\left(240 \mathrm{~mA} \cdot v \cdot t_{p}\right)$, where $t$ is the NIC idle time.

\section{MDR Metric}

An energy aware metric can be considered such as explained in [15]. However, in this section we recall just the cost function considered in our Multipath DSR extensions. It is the Minimum Drain Rate (MDR) and it permits a cost associated with a node to be calculated as referred to in (1):

$$
C_{i}=\frac{R B P_{i}}{D R_{i}}
$$

where $R B P_{i}$ denotes the residual battery energy at node $n_{i}$, and it indicates when the remaining battery energy of node $n_{i}$ is exhausted, i.e., how long node $n_{i}$ can keep up with routing operations with current traffic conditions based on the residual energy. $D R_{i}$ is calculated by utilizing the well-known exponential weighted moving average method (see (2)) applied to the drain rate values $D R_{\text {old }}$ and $D R_{\text {curr }}$, which represent the previous and the current calculated values.

$$
D R_{i}=\alpha \cdot D R_{\text {old }}+(1-\alpha) \cdot D R_{\text {curr }}
$$

The maximum lifetime of a given path $r_{p}$ is determined by the minimum value of $C_{i}$ over the path, that is $L_{p}=\min _{\forall n_{i} \in r_{p}} C_{i}$.

The Minimum Drain Rate (MDR) mechanism is based on selecting the route $r_{M}$, contained in the set $R$ of all possible routes between the source and the destination nodes, that presents the highest maximum lifetime value, that is: $r_{M}=r_{p}=\min _{\forall r_{i} \in R} L_{i}$. 


\section{IV.MEA-DSR Protocol}

To have all possible paths between a source-destination pair, the destination replies to all ROUTE REQUESTs (RREQ)s that arrive, and the source stores all the paths of received ROUTE REPLY (RREP)s. Among all the stored paths, only node-disjoint routes are considered in accordance with [8]. The paths are ordered not just by path length (minimum hop count metric), but by an energetic metric. This energetic metric is computed while the RREP crosses the network from destination to source and it is stored in the routing table at source. The value of this metric is updated for all stored paths to avoid using an incorrect value that could compromise the performance of the protocol.

\section{Route Discovery Mechanism}

When a node $s$ originates a new packet destined to some other node $d$, it places in the header of the packet a source route giving the sequence of hops that the packet should follow on its way to $d$. Normally, $s$ will obtain a suitable source route by searching its Route Cache routes previously learned, but if no route is found in its cache, it will initiate the Route Discovery protocol to dynamically find a new route to $d$.

To initiate the Route Discovery, $s$ transmits a RREQ message as a single local broadcast packet, which is received by (approximately) all nodes currently within its wireless transmission range.

When another node receives a RREQ, if it is $d$, it returns a message RREP to $s$. Otherwise, if this node is an intermediate node it forwards the RREP if it has no path toward destination in its own cache. If an intermediate node knows a route to destination, replies to $s$ with a RREP, in which it copies the records stored in the RREQ and appends the rest of the path that is in its cache.

To have energy information, in accordance with [15], we have introduced a new field in DSR packet, such as referred in fig.1. The field $C_{i}$ contains the cost function value. Before the destination sends back a RREP, it inserts in field $C_{i}$ its cost function value. While the RREP is propagating to source, each node calculates its $C_{i}$, checks it with the value contained in RREP and, if it is necessary, replaces $C_{i}$ contained in RREP with its one.

In source Route Cache the path will be stored as an entry $\left\langle\right.$ path, $C_{i}>$, where the path contains all the nodes $I D$ and $C_{i}$ is the cost function of the entire path. The paths to the same destination are ordered from the "best" to the "worst"; where the best stay owing to their greater energy-efficiency according with the chosen energy metric. In this case as a first step more energy metrics were considered such as in [15] and the best performers were selected.

Multiple paths store in the cache are only node-disjoint and this means that the routes have no nodes or links in common. Disjoint routes offer certain advantages 
over non-disjoint routes. For instance, non-disjoint routes may have lower aggregate resources than disjoint routes, because non-disjoint routes share links or nodes. In principle, node-disjoint routes offer the most aggregate resources, because neither links nor nodes are shared between the paths. Disjoint routes provide also higher fault-tolerance. When using non-disjoint routes, a single link or node failure can cause multiple routes to fail. In node or link disjoint routes, a link failure will only cause a single route to fail.

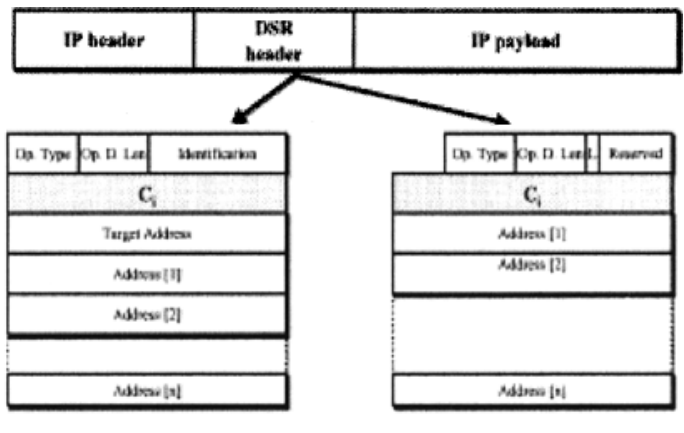

Route Request

Route Reply

Figure 1: Modified DSR PDU

The number of node-disjoint paths is smaller than non-disjoint paths, but if a node is shared among different routes, energy consumption balancing decreases.

\section{Update Mechanism}

As stated, when a source receives a RREP it stores path information and $C_{i}$ value. Since nodes receive and transmit packets all time, they consume their energy, so the value of $C_{i}$ of each node changes in time and information in source route cache become inconsistent with the real conditions of the network.

An update mechanism is needed because the following situation can occur. In cache there is a path that seems to have sufficient energy to support a given traffic load, while really this path will run down soon if high data traffic crosses through itself. The Update Mechanism consists in a periodic updating of the cache through probe packets that are sent on all active paths of the set $R$.

Periodically all the nodes send a unicast probe request packet, called RREQPROBE, for all paths $p \in R$ in cache. The RREQ-PROBE is similar to a RREQ packet, but it is not sent in broadcast, because there is already a path to destination. When a RREQ-PROBE arrives at destination, a RREP-PROBE is sent back. The RREP-PROBE is a simple RREP with a set flag probe. Since the RREPPROBE is a kind of RREP, it contains the destination $C_{i}$ value. When it is received by an intermediate node, the node calculates its $C_{i}$, it checks this value 
with the value contained in the RREP-PROBE and, if it is necessary, it replaces the current cost with its calculated value. When the source receives an RREPPROBE it simply updates information in its cache. This simple mechanism will allow the update of the set $R$ of the active path, erasing the path from $R$ that drains their energy too much.

\section{Performance Evaluation}

In this section, let DSR indicate the original Dynamic Source Routing Protocol, DSR-MDR is the abbreviation for DSR with Minimum Drain Rate (MDR). MDSR indicates multipath DSR implemented in [5], MDSR-MDR is for MDSR with MDR and MEA-DSR-MDR is the version of MEA-DSR with MDR metric and with Update Mechanism. The variant of MEA-DSR with other energy aware metrics is indicated as MEA-DSR-(energy metric). For example in the case of MBCR metric use the protocol is called MEA-DSR-MBCR such as referred in the legend of simulation graphics.

\section{Simulation Scenario}

This study is concentrated on evaluating average energy among nodes over time and the connection expiration time. Each simulation has a duration of $451 \mathrm{sec}-$ onds. During each simulation, 8 constant bit rate (CBR) connection were generated, producing 4 packets/seconds with a packet size of 64 bytes. The transmission range used is fixed at 250 meters for all nodes.

The "random waypoint" model was used to simulate node movement. The motion is characterized by two factors: the maximum speed and the pause time. Each node starts moving from its initial position to a random target position selected inside the simulation area. When a node reaches the target position, it waits for the pause time, then it selects another random target location and moves again.

The ns- 2 energy model was modified to allow the measuring of the battery energy.

\section{Comparison among different metrics (MBCR, MMBCR, MDR)}

This sub-section compares the performance of the MDR mechanism against MBCR (Minimum Battery Cost Routing) and MMBCR (Min-Max Battery Cost Routing) mechanisms (details of these metrics can be found in $[14,15]$ and an extensive simulation under DSR can be found in [6]) using ns-2 simulator with the CMU wireless extension. The MEA-DSR-MDR protocol was considered. A network consisting of 40 mobile nodes distributed over a $1850 \times 1850$ meters area is considered. MEA-DSR was used to evaluate the behaviour of the MBCR, MMBCR and MDR mechanisms when all the nodes maintain their initial position for the simulations duration and when all the nodes move around the network. Figure. 2 shows the comparison of average energy among all the nodes remaining at the end of the simulation for different mechanisms.

The MEA-DSR-MBCR approach attempts to maximize the lifetime of each 
host. Since cost function of the path is the sum of all the cost functions of the nodes, a route containing nodes with lower remaining battery capacity may still be selected if the other nodes along other routes have a greater remaining capacity; so the use of run-down nodes is not prevented, therefore in the network there will be nodes with more residual energy and nodes that are not alive, so the average energy will be a lower value.

The MEA-DSR-MMBCR approach tries to distribute evenly the energy consumption among nodes by using their residual battery capacity. However, since it allows nodes to accept all the connection requests if they temporarily have enough battery regardless of current traffic condition, the nodes will eventually experience lack of battery. The absence of some particular nodes owing to the traffic overload, forces the current connection to attempt to establish a new route. Therefore MEA-DSR-MMBCR suffers from the short lifetime of connections. On the other hand MEA-DSR-MDR seems to use longer routes among a few paths even in the sparse network to balance energy consumption among nodes. As some nodes die over time, the total number of possible routes between the source and destination nodes decreases. Moreover, the node movement allows new routes to appear.

Therefore, MEA-MMBCR and MEA-MDR can balance traffic by alternating the use of existing routes with different hops.

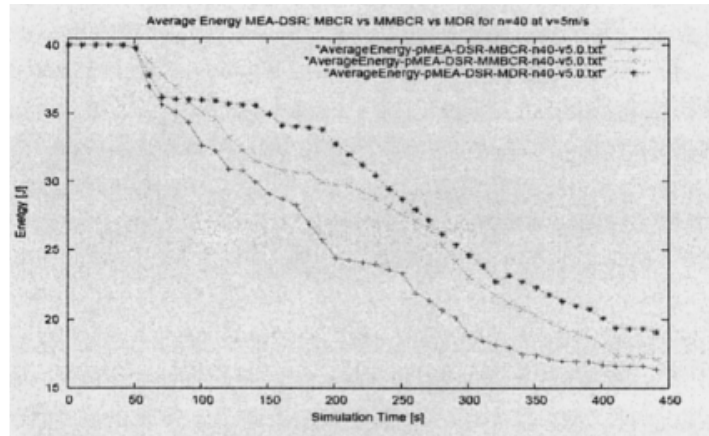

Figure 2: Average Energy MEA-DSR: $\mathrm{MBCR}-\mathrm{MMBCR}-\mathrm{MDR} v=5 \mathrm{~m} / \mathrm{s}$

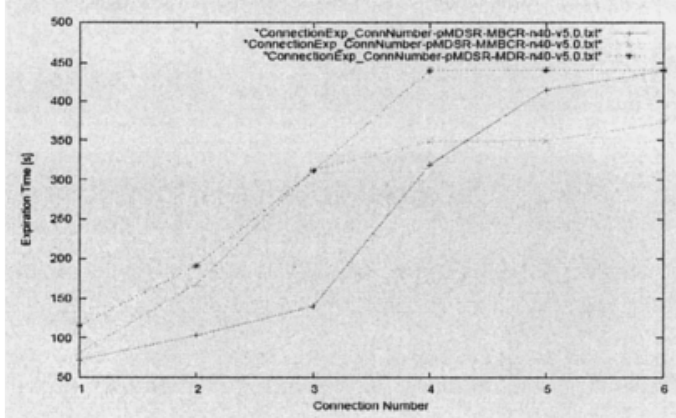

Figure 3: Expiration of Connections MEA-DSR: MBCR $-\mathrm{MMBCR}-\mathrm{MDR}$ with v=5m/s. 
The MEA-DSR-MDR approach can properly extend the lifetime of nodes and of connections (see Figure.3) by evenly distributing the energy expenditure among nodes. It avoids the over-dissipation of specific nodes by taking into account the current traffic condition and by utilizing the drain rate of the residual battery capacity. The main goal of MEA-MDR is to avoid the over-dissipation of energy at critical nodes in order to extend the lifetime of connections. After all those simulation analysis, MDR is chosen as the energy metric of the Multipath DSR. With the addition of round robin scheduling scheme and the cache update through probe packets, the protocol is called MEA-DSR-MDR .

\section{MEA-DSR-MDR and Update mechanism evaluation}

Figure 4 illustrates the lifetime of connections is longer for a value of 20 seconds. Updating every 20 seconds means having more current information instead of updating in 40 seconds, but it also means inserting in the network almost double of the probe packets. The difference between a timer value of 20 seconds and 40 is more evident for low mobility (Figure. 4) than high mobility (Figure. 5). The reason is that high mobility takes more frequent route breakage and this determines an implicit updating of cache made by new route discovery processes.

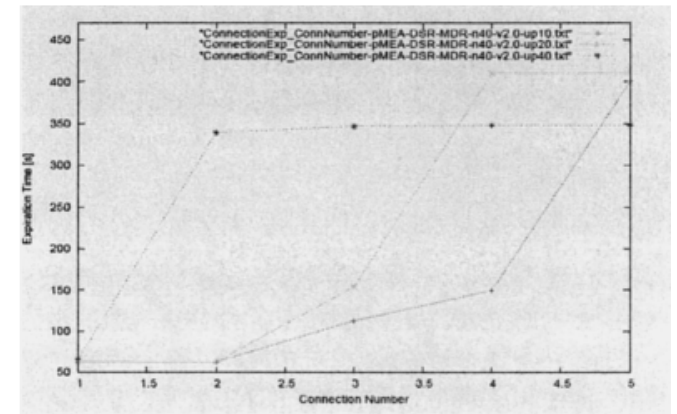

Figure 4: Expiration of connections MEA-DSR: update timer 10-20-30 $\mathrm{sec}$ and $\mathrm{v}=2 \mathrm{~m} / \mathrm{s}$.

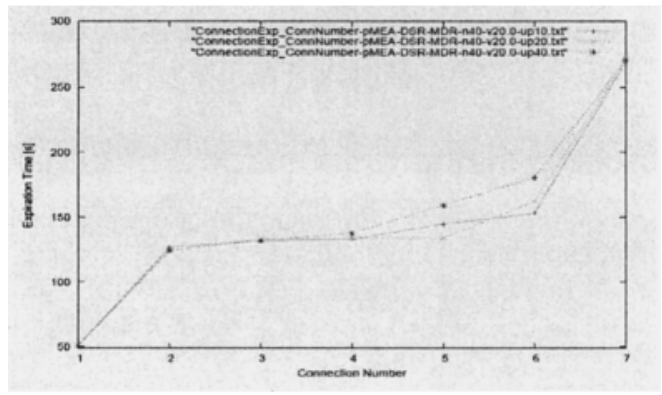

Figure 5: Expiration of connections MEA-DSR: update timer $10-20-30 \mathrm{sec}$ and $\mathrm{v}=20 \mathrm{~m} / \mathrm{s}$ 


\section{Comparison of MEA-DSR-MDR with DSR and MDSR}

Up to now it has been discussed how to implement MEA-DSR-MDR, the effect of the update rate and what energy metric is more efficient. Now DSR-MDR, MDSR-MDR, MEA-DSR-MDR are also considered for comparison purpose. MDSR has been implemented with the addition of probing packets to test and update multiple routes state and round robin scheduling for sending data packets over multiple routes.

Multiple paths between source and destination node pairs can be used to compensate for the dynamic and unpredictable nature of ad hoc networks. Spreading the traffic among multiple routes can improve load balancing, alleviate congestion and bottlenecks, and prolong nodes and connections lifetime, thereby saving more energy .

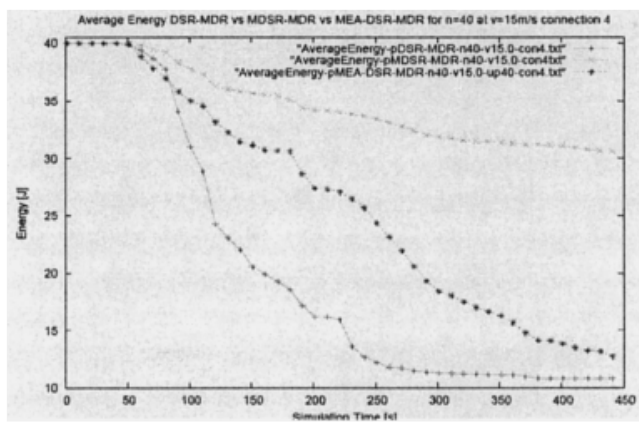

a)

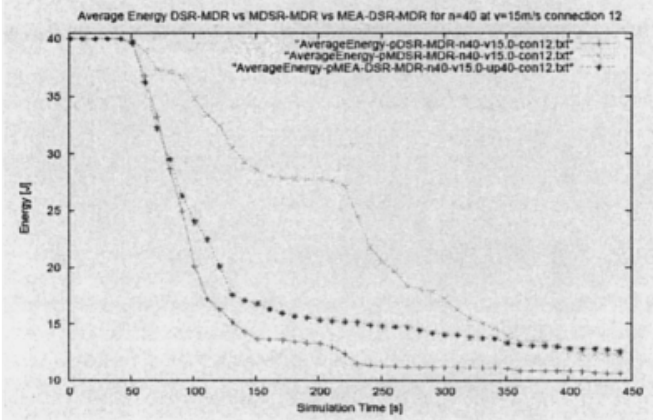

b)

Figure 6: Average Energy DSR MDSR MEA-DSR v=15m/s a) 4 connection and 64 bytes packet length; b) 12 connection and 64 bytes packet length;

For high mobility (Figure 6), the update mechanism penalizes MEA-DSRMDR compared with MDSR-MDR, this is caused by the amount of unnecessary probe packets sent on the network. The overhead is bigger for MEA-DSR-MDR than MDSR-MDR. It is truth for all considered speeds, but its weight is more evident for high mobility. The nodes movement determines frequent route breakages and forces new Route Discovery processes. More rapidly the nodes move, more 
frequently route discoveries start causing overhead increase. In MEA-DSR-MDR overhead caused by probe packets must be added. So in MEA-DSR, nodes spend more energy because they transmit and receive more packets.

On other hand, delivery data packet increases and end to end delay decreases in MEA-DSR-MDR with respect to MDSR-MDR such as shown in Table I for a probing time of $20 \mathrm{~s}$. However, the control overhead is increased for the probe packet forwarding.

Table 1: Performance Evaluation of Unipath and Multipath Routing with MDR

\begin{tabular}{|l|c|c|c|}
\hline \multicolumn{1}{|c}{ METRIC } \\
\hline Overhead (\%bytes) & DSR & MDSR & MEA-DSR \\
\hline E2E delay (ms) & 2.4 & 2.8 & 3.5 \\
\hline Data Packet Delivery ratio (\%) & $20 \mathrm{~ms}$ & $14 \mathrm{~ms}$ & $10 \mathrm{~ms}$ \\
\hline
\end{tabular}

\section{VI.CONCLUSIONS}

A novel energy aware multipath routing protocol has been proposed (MEA-DSR). It has been integrated with different energy metrics such as MBCR, MMBCR and MDR. This latter metric has proved the best choice to apply on the MDSR protocol. Simulation results showed how a simple round robin mechanism permits an energy load balancing and a fair distribution of the energy, extending the connection time. Also a periodical Update Mechanism has been tested. This permits one to update the source cache but it can introduce more overhead on the network. For low mobility this mechanism can offer some advantages by reducing the E2E delay and increasing the data packet delivery ratio.

\section{ACKNOWLEDGEMENT}

We thank Polytechnic University of Valencia and, in particular, Prof. Juan Carlos Cano and Prof. Pietro Manzoni for their useful suggestions and explanations provided about the MDR metric.

\section{REFERENCES}

[1] E. Royer and C.-K. Toh, "A Review of Current Routing Protocols for Ad Hoc Mobile Wireless Networks," IEEE Personal Comm.Magazine, vol. 6, no. 2, Apr. 1999.

[2] S. Mueller, R.P.Tsang, D.Ghosal, "Multipath Routing in Mobile Ad Hoc Networks: Issues and Challenges," Invited paper in Lecture Notes in Computer Science, Edited by Maria Carla Calzarossa and Erol Gelenbe, 2004.

[3] M.R.Pearman, Z.J.Haas, P.Sholander, S.T. Tabrizi, "On The Impact of Alternate Path Routing for Load Balancing in Mobile Ad Hoc Networks," in Proc. of 1 ACM International Symposium on Mobile Ad Hoc networking \& Computing, Boston, Massachussets, pp.3-10, 2000.

[4] Lee, S.-J., Gerla, M., "Split Multipath Routing with Maximally Disjoint Paths in Ad Hoc Networks," IEEE International Conference on Communications, Vol. 10 (2001)

[5] Marina, M.K., Das, S.R, "On-demand Multipath Distance Vector Routing in Ad Hoc Networks," Proceedings of ICNP, 2001.

[6] M.Fotino, et al., "Evaluating Energy-aware Behaviour of Proactive and Reactive Routing Protocols for Mobile Ad Hoc Networks," in SPECTS'07, 16-18 July, San Diego, CA, USA. 
[7] Z., Krishnamurthy, S.V., Tripathi, S.K, "A Framework for Reliable Routing in Mobile Ad Hoc Networks," IEEE INFOCOM (2003).

[8] A. Mohammad Abbas, B.N.Jain, "Mitigating Overheads and Path Correlation in Node-Disjoint Multipath Routing for Mobile Ad hoc Networks" in Proc. of Ist lEEE COMSWARE, New Delhi, January 8-12, 2006.

[9] C.Chen, W. Wu, Z. Li "Multipath Routing Modelling in Ad Hoc Network" IEEE ICC, 16-20 May 2005.

[10] V.Loscrì, F. De Rango, S. Marano, "Ad Hoc On Demand Distance Vector Routing (AOMDV) over a Distributed TDMA MAC Protocol for QoS support in Wireless Ad Hoc Networks: Integration Issues and Performance Evaluation," ETT Journal, Vol.18, Issue 2, 2007.

[11] J. Gomez, A.T. Campbell, M. Naghshineh, and C. Bisdikian, "Conserving Transmission Power in Wireless Ad Hoc Networks," Proc. Ninth Int'l Conf. Network Protocols, 2001.

[12] L. Feeney and M. Nilsson, "Investigating the Energy Consumption of a Wireless Network Interface in an Ad Hoc Networking Environment," Proc. IEEE INFOCOM, 2001.

[13] S. Singh, M. Woo, and C.S. Raghavendra, "Power-Aware with Routing in Mobile Ad Hoc Networks," Proc. Fourth Ann. ACM/IEEE Int'l Conf. Mobile Computing and Networking, 1998.

[14] C.-K. Toh, "Maximum Battery Life Routing to Support Ubiquitous Mobile Computing in Wireless Ad Hoc Networks," IEEE Comm. Magazine, June 2001

[15] D.Kim, et al., "Routing Mechanisms for Mobile Ad Hoc Networks Based on the Energy Drain Rate" IEEE Trans.on Mob. Comp, vol. 2, no. 2, Apr-June 2003

[16] J. Li, D.Cordes and J. Zhang "Power - Aware Routing Protocols in Ad Hoc Wireless Network" IEEE Wireless Communication - December 2005

[17] W. Kim, X. Yu-long, C. Guo-liang and W. Ya-feng, "Power-aware On-demand Routing Protocol for MANET," in Proc. of the 24th Int. Conf, on Distributed Computing Systems, March 2004.

[18] C.F. Chiasserini and R. R. Rao, "Routing Protocols to Maximize Battery Efficiency," Proc. 21st Century Military Commun. Conf., vol. 1, Oct. 2000, pp. 496-500. 